\title{
Uniportal video-assisted thoracic surgery in the treatment of pleural empyema
}

\author{
Mahmoud Ismail ${ }^{1 \#}$, Dania Nachira ${ }^{2 \#}$, Elisa Meacci ${ }^{2}$, Gian Maria Ferretti ${ }^{2}$, Marc Swierzy ${ }^{1}$, Julianna \\ Paulina Englisch $^{1}$, Ramin Raul Ossami Saidy ${ }^{1}$, Svea Faber ${ }^{1}$, Maria Teresa Congedo ${ }^{2}$, Marco Chiappetta ${ }^{2}$, \\ Leonardo Petracca Ciavarella ${ }^{2}$, Stefano Margaritora ${ }^{2}$, Jens C. Rueckert ${ }^{1}$ \\ ${ }^{1}$ Competence Center of Thoracic Surgery, Department of Surgery, Charité - Universitätsmedizin Berlin, Berlin, Germany; ${ }^{2}$ Department of General \\ Thoracic Surgery, Fondazione Policlinico Universitario “A. Gemelli”, Rome, Italy \\ Contributions: (I) Conception and design: M Ismail, D Nachira; (II) Administrative support: JC Rueckert, S Margaritora; (III) Provision of study \\ materials or patients: E Meacci, M Swierzy, MT Congedo, M Chiappetta, L Petracca Ciavarella; (IV) Collection and assembly of data: GM Ferretti, \\ M Swierzy, JP Englisch, S Faber, RR Ossami Saidy; (V) Data analysis and interpretation: D Nachira, M Ismail; (VI) Manuscript writing: All authors; \\ (VII) Final approval of manuscript: All authors. \\ "These authors contributed equally to this work. \\ Correspondence to: Mahmoud Ismail, MD. Competence Center of Thoracic Surgery, Department of Surgery, Charité- Universitätsmedizin Berlin, \\ Charitéplatz 1, Berlin 10117, Germany. Email: mahmismail@gmail.com.
}

Background: The efficacy of video-assisted thoracic surgery (VATS) in the treatment of pleural empyema has recently been proven. Till today, very few works evaluated the role of uniportal-VATS (U-VATS) approach in the treatment of pleural empyema even if it currently represents the most innovative and less invasive thoracoscopic approach. We report our experience with U-VATS in the treatment of pleural empyema.

Methods: A retrospective bicentric analysis of 35 consecutive patients who underwent surgical treatment of stage II and stage III pleural empyema was performed, from January 2015 to May 2017.

Results: The mean age of patients was $57.26 \pm 18.29$ years and $54.3 \%$ of them were males. In $85.7 \%$ of the cases, empyema was related to a complicated parapneumonic effusion; in only 5 cases it was a post-surgical consequence. All patients were treated with broad-spectrum antibiotics and subsequent target therapy for $14.62 \pm 21.76$ days prior to operation and 23 patients needed the placement of a chest tube. Twenty patients (57.1\%) presented with stage III, 11 patients (31.4\%) stage II and 4 patients (11.4\%) stage I empyema. Complete debridement and decortication were obtained in all patients through U-VATS approach and no conversion or further access was needed for any reason. No major complication was recorded. Only 2 cases of trapped lung were not responsive to surgical treatment. At a mean follow-up of $247.42 \pm 306.29$ days, 33 patients $(94.3 \%)$ were alive with no recurrence, 2 patients died for causes unrelated to the operation.

Conclusions: According to our experience, we consider U-VATS as an adequate procedure in the treatment of "stages II and III" empyemas when the necessary surgical expertise has been achieved. Indeed, U-VATS permits an easier performance and complete debridement and decortication, with a very low risk for conversion and excellent postoperative outcomes in terms of less pain, fast recovery and cosmetic results.

Keywords: Pleural empyema; uniportal video-assisted thoracic surgery (U-VATS); decortication

Submitted Feb 15, 2018. Accepted for publication Apr 24, 2018.

doi: $10.21037 /$ jtd.2018.05.10

View this article at: http://dx.doi.org/10.21037/jtd.2018.05.10 


\section{Introduction}

Pleural empyema is defined as the presence of purulent fluid in the pleural cavity.

It is due to pleural space infection, as a result of a postbacterial pneumonia in the majority of cases, and it is associated with a significant morbidity and mortality of $2-30 \%$ (1). Incidence and prevalence of pleural empyema vary depending on geography, aetiology, age and host immune status. Aetiology is determinant for clinical therapeutic choices while the surgical approach to pleural empyema is determined by its evolutive stage and it is required in the $36-65 \%$ of patients (2). In fact, according to its radiological [X-ray, computed tomography (CT) scan and ultrasonography] features, empyema is classified into three stages (3):

* Stage I: "Parapneumonic effusion", with an increase in pleural effusion;

* Stage II: "Fibrinopurulent stage" with loculations of pleural fluid and fibrinous septa formation;

* Stages III: "Chronic organizing stage" with scar adhesions and progressive constriction unto incarcerated lung.

While in 'stage I' the suggested therapy is based on positioning of chest tube drainage and antibiotics as advised by the Guideline Group of the British Thoracic Society (BTS) (2), in 'stages II and III' surgery is applied to perform debridement and/or decortication with the aim to achieve a complete and quicker lung re-expansion and eliminate all the purulent organized collections that could not be removed otherwise.

Demonstrated advantages of an early surgical approach in the last two stages of pleural empyema are a reduction of hospital costs, morbidity and mortality (4).

Nowadays, the most frequently practiced surgical techniques are video-assisted thoracic surgery (VATS) and thoracotomy. The first plays a bigger role in both 'stages II and III', with better outcomes if compared with thoracotomy $(2,5)$. The second is still performed in a high number of cases in 'stage III' because there is an important conversion rate from VATS technique when a more complete decortication is needed $(2,5)$.

The application of the uniportal-VATS (U-VATS) approach for the treatment of pleural infection has not yet been well defined up to now.

We report our experience with U-VATS in the treatment of pleural empyema, mainly in II and III stages, for critically evaluating the safety, effectiveness and clinical outcomes of the technique.

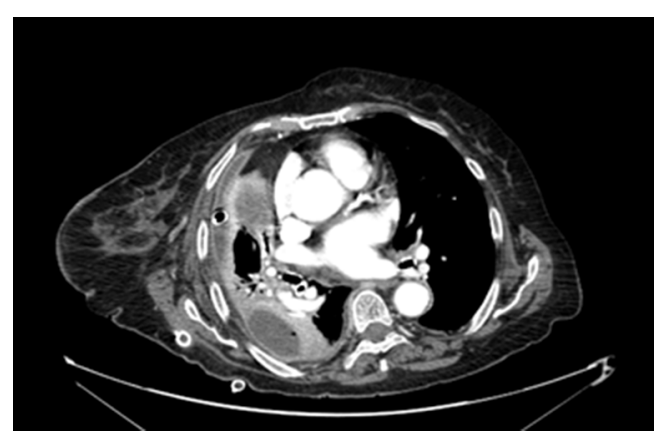

Figure 1 Preoperative CT scan of a III stage empyema of the right chest cavity.

\section{Methods}

From January 2015 to May 2017, the prospectively collected clinical data of 35 consecutive patients who underwent surgical treatment for pleural empyema, were retrospectively reviewed in a multicentric study performed by the departments of Thoracic Surgery of Charité University Hospital (Berlin, Germany) and Fondazione Policlinico "A. Gemelli" (Rome, Italy).

All patients underwent thoracoscopic pleural toilette, debridement and decortication in U-VATS approach. All patients signed an informed consent before the operation for the treatment of their clinical data.

A first level approach to diagnosis was performed through anamnesis, clinical examination, blood sample analysis with phlogosis indices panel (white cells count, serum C-reactive protein and serum procalcitonin), chest $\mathrm{X}$-ray and ultrasonography. The second level examination to confirm our clinical diagnosis was a chest CT scan (Figure 1). All patients were treated with broad spectrum antibiotic therapy, waiting for microbiological results (analysis of bronchoalveolar lavage or pleural effusion samples) necessary to set a target therapy up.

When necessary (pleural effusion amount $>1,000-1,500 \mathrm{~mL}$ without radiological-ultrasound or CT-features of a gelatinous or organized fluid collection with several septa) a chest tube drainage $(20,24$ or $28 \mathrm{Fr}$ thoracic trocar catheter) was inserted (usually a single chest drain insertion was made in the biggest pocket in case of multiloculated pleural effusion, using ultrasound guidance) to allow an improvement of the respiratory functions and collect pleural effusion samples to perform microbiological and chemical examinations to set targeted antibiotic therapy.

If the following clinical and radiological examinations showed the failure of medical therapy with persistence of 

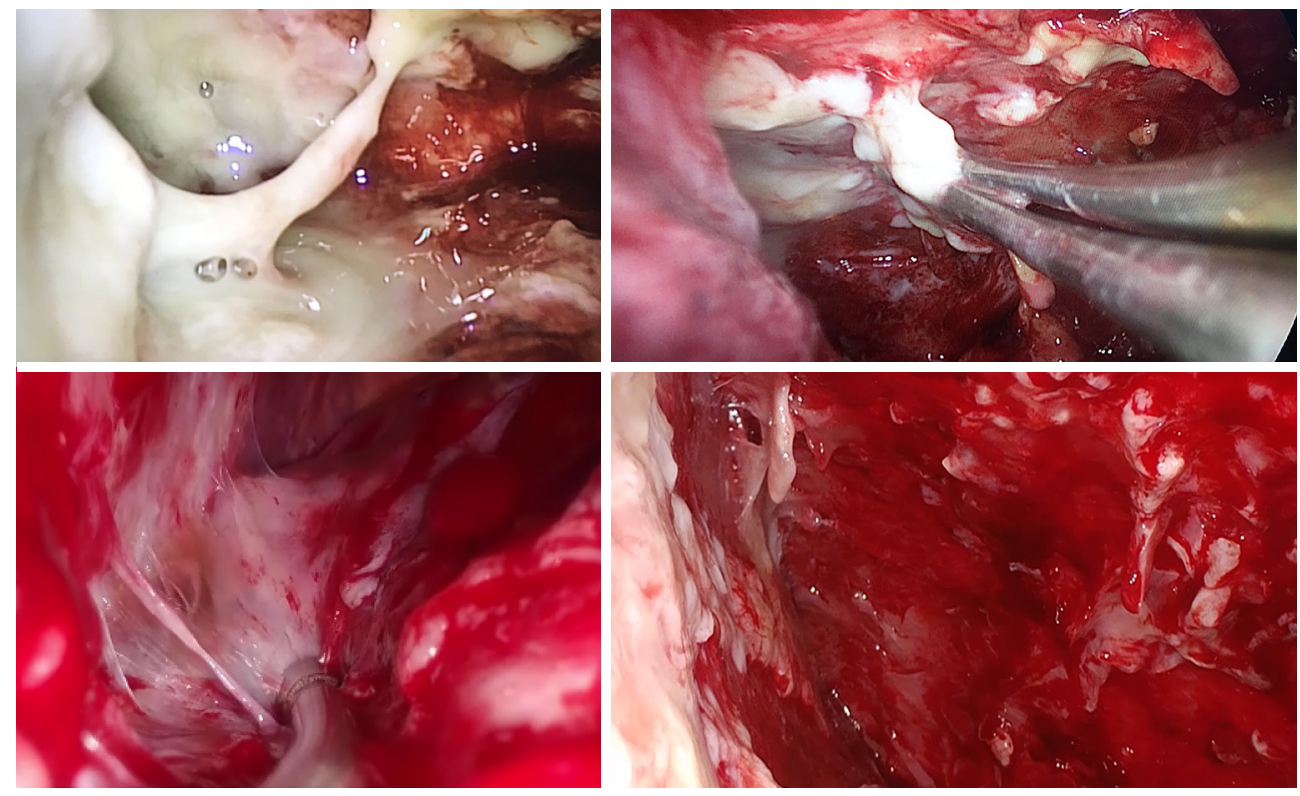

Figure 2 Intraoperative findings and uniportal-VATS debridement of empyema septa. VATS, video-assisted thoracic surgery.

septic status, trapped lung or several loculations of pleural fluid and presence of fibrinous septa (stages II and III), patients were sent to U-VATS surgical treatment, after multidisciplinary consult.

\section{U-VATS technique}

All procedures were performed under general anaesthesia and single lung ventilation, using a double-lumen endotracheal tube. The patient was then put in lateral decubitus position with the arms flexed and stretched towards the head (6). A single 3-4 cm muscle-sparing incision (without cutting muscular fibers, only spreading the insertions of serratus anterior muscle on the ribs and making a fascial slide of surrounding muscles) was performed at the $5^{\mathrm{TH}}$ intercostal space on the mid axillary line. Incision widening and protection for the introduction of a $10 \mathrm{~mm} 30^{\circ}$ thoracoscope and endoscopic instruments was obtained with a wound protector.

The operation proceeded with septal rupture, debridement and removal of all adhesions and inflammatory effusions from the diaphragmatic and parietal pleura to apex of chest cavity with the aim to create a unique pleural cavity (without septa and loculations) and to restore the physiological movement of the lung (Figure 2). All instruments used (suction instrument and decortication grasper) had a long and curved shaped, with a dual pivot point design for enabling the simultaneous use of multiple instruments through the same access.

During this step, microbiological and histological samples were taken. Multiple washings with warm physiological solution were carried out to eliminate all the residual effusion and organized pus from the visceral pleura with minimal parenchymal injury.

Decortication was achieved through the removal of the thickened visceral pleura or through its multiple incisions with an electrocautery device (Figure 3). Under thoracoscopic control, lung inflation was performed to evaluate the efficacy of decortication through parenchymal re-expansion.

An extrapleural paravertebral intercostal nerve block was always performed, infiltrating $3 \mathrm{~mL}$ of ropivacaine $(4.75 \mathrm{mg} / \mathrm{mL})$ in $3-4$ intercostal spaces above and below the incision, under endoscopic view (6).

The procedure ended when a full lung re-expansion was accomplished and with the positioning of one or two (in case of severe empyema that required an extensive visceral and parietal pleural decortication) chest tube drainages (20, 24 or $28 \mathrm{Fr})$ through the same incision (6).

Timing of chest tube removal depended on surgical reasons (ending of air leak or when drained fluid was clear and the amount in 24 hours was less than $200 \mathrm{~mL}$ ), clinical reasons (apyrexia, decreased levels of inflammatory mediators) and radiological improvement (complete lung 


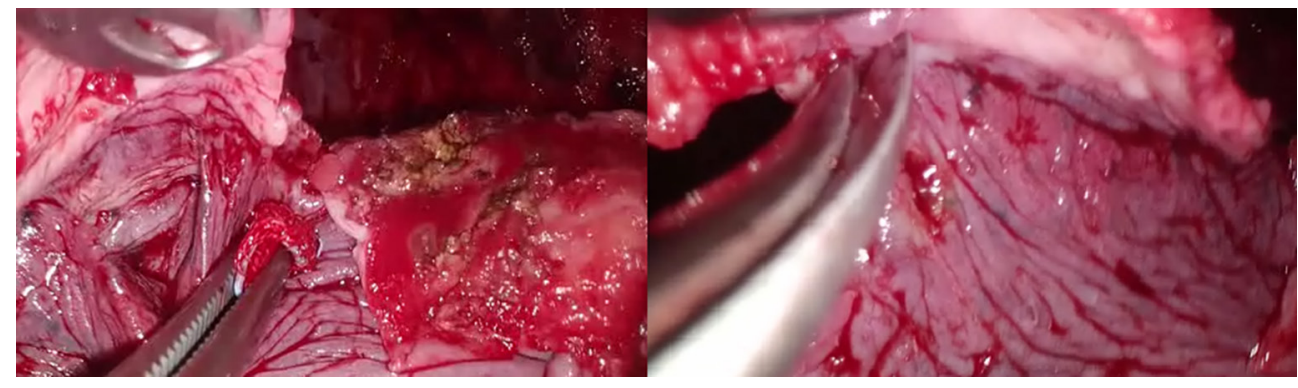

Figure 3 Uniportal-VATS decortication of visceral pleura. VATS, video-assisted thoracic surgery.

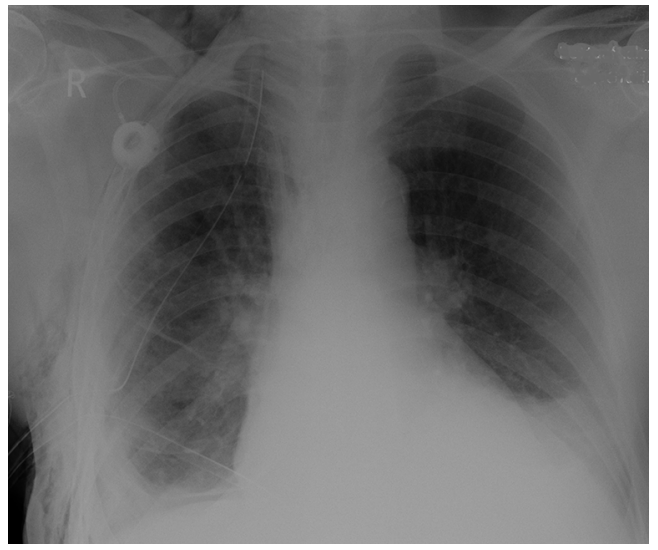

Figure 4 Postoperative X-ray of the same patient, of whom preoperative CT and intraoperative findings are shown in Figure 1 and 2 , respectively.

re-expansion, Figure 4).

\section{Statistical analysis}

Statistical analysis was performed using PASW Statistics for Windows, Version 18.0 (SPSS Inc., Chicago, IL, USA). Continuous variables were expressed as mean and standard deviation.

Pearson $\chi^{2}$ test and Fischer's exact test were used to compare discrete variables and Student's $t$-test to compare means between two continuous variables. A P value less than 0.05 was considered statistically significant.

\section{Results}

The mean age of patients was $57.26 \pm 18.29$ [25-86] years and the $54.3 \%$ (19 patients) were males.

The main demographical and clinical characteristics of the patients are reported in Table 1 .

In the most part of the cases $(85.7 \%, 30$ patients), empyema was related to a complicated parapneumonic effusion; only in 5 cases it was a post-surgical consequence.

High fever was the main symptom in the $62.9 \%$ of the series (22 cases), 10 (28.6\%) patients presented cough or dyspnoea and $3(8.6 \%)$ only chest pain at the admission.

All patients were treated with broad-spectrum antibiotic therapy and subsequent target therapy (after determination of responsible germs by bronchoalveolar lavage in 9 cases or analysis of pleural effusion in 23 cases) for $14.62 \pm 21.76$ days prior to operation. In the same period, in 23 patients a chest tube was placed for evacuating pleural effusion, making microbiologic insights and evaluating lung re-expansion. No fibrinolytic therapy had been administered. Among patients treated with chest tube insertion, 19 patients showed a trapped lung.

Almost the whole series ( $97.1 \%$ of patients) underwent a preoperative CT-scan for the assessment of pulmonary condition.

Twenty patients (57.1\%) presented a III stage empyema, 11 patients $(31.4 \%)$ a II stage and 4 patients $(11.4 \%)$ a I one.

The main etiologic agents were: Klebsiella pneumonia, Streptococcus constellatus, Chlamydia pneumonia and Mycobacterium tuberculosis.

The mean operation time for U-VATS approach was $128.29 \pm 66.51 \mathrm{~min}$. A complete debridement and decortication were obtained in all patients and no conversion or further access was needed for any reason.

No air-leakage $>5$ days was recorded. Minor complications (Table 2) were described in $8(22.8 \%)$ patients. The intraoperative and 30-day mortality were null.

The last drainage was removed after $8.91 \pm 7.01$ days and patients were discharged after $15.06 \pm 8.98$ days.

In $94.3 \%$ of patients (33 cases) there was a good 
Table 1 Demographical and clinical characteristics of the patients

\begin{tabular}{|c|c|}
\hline Characteristics & $\mathrm{N}=35(\%)$ \\
\hline Age (years) & $57.26 \pm 18.29$ \\
\hline \multicolumn{2}{|l|}{ Gender } \\
\hline Male & $19(54.3)$ \\
\hline Female & $16(45.7)$ \\
\hline Smoking & $6(17.1)$ \\
\hline COPD & $5(14.3)$ \\
\hline Heart disease & $9(25.7)$ \\
\hline Arterial hypertension & $18(51.4)$ \\
\hline Diabetes & $4(11.4)$ \\
\hline \multicolumn{2}{|l|}{ ASA score } \\
\hline 2 & $13(37.1)$ \\
\hline 3 & $21(60.0)$ \\
\hline 4 & $1(2.9)$ \\
\hline Preoperative pulmonary infections & $30(85.7)$ \\
\hline Preoperative white blood cell count & $14,300 \pm 7,445$ \\
\hline Preoperative C-reactive protein ( $<5 \mathrm{mg} / \mathrm{L})$ & $173.32 \pm 99.62$ \\
\hline Preoperative procalcitonin $(<0.5 \mathrm{ng} / \mathrm{mL})$ & $1.22 \pm 2.19$ \\
\hline \multicolumn{2}{|l|}{ Empyema stage } \\
\hline I & $4(11.4)$ \\
\hline II & $11(31.4)$ \\
\hline III & $20(57.1)$ \\
\hline Operating time (min) & $128.29 \pm 66.51$ \\
\hline \multicolumn{2}{|l|}{ Side } \\
\hline Right & $15(42.9)$ \\
\hline Left & $20(57.1)$ \\
\hline \multicolumn{2}{|l|}{ Number of chest tubes } \\
\hline One & 24 (68.6) \\
\hline Two & $11(31.4)$ \\
\hline
\end{tabular}

COPD, chronic obstructive pulmonary disease.

Table 2 Minor complications

\begin{tabular}{lc}
\hline Complications $[\mathrm{N}=8(22.8 \%)]$ & $\mathrm{n}(\%)$ \\
\hline Atelectasis & $2(5.7)$ \\
Anemia & $6(17.1)$ \\
Air-leakage $>5$ days & $0(0)$ \\
\hline
\end{tabular}

re-expansion of the lung, with only 2 cases of trapped lung not responsive to surgical treatment. The only significant risk factor for postoperative trapped lung was the presence of a tracheotomy before the operation (P: 0.015).

All phlogosis indexes improved significantly after the operation (Table 3).

No correlation was found between the stage of the empyema and chest tube duration, incidence of trapped lung or complications. Only a trend towards statistical significance between the number of chest tubes ( 2 or 1 ) and the empyema stage (P: 0.09) was noted. However, the number of drainages didn't influence in a significant way the chest tube duration (P: 0.22).

At a mean follow-up of $247.42 \pm 306.29$ days, 33 patients $(94.3 \%)$ were alive with no recurrence, 2 patients died for causes unrelated to the operation.

After U-VATS approach, the level of pain was measured on VAS scale once a day (at the same time in the morning before any painkiller assumption), every day after the operation until 5-6 hours after the chest tube removal.

The postoperative level of pain (Table 4) was found to be very low (mean value measured on VAS scale in I postoperative day: $1.83 \pm 1.53$ ) and the mean duration of pain was $2.63 \pm 2.23$ days, with an almost total resolution after chest tube removal (mean value of pain on VAS scale: $0.33 \pm 0.92$ ).

No correlation between post-operative pain and factors like chest tube duration, number of drainages, age, days of painkiller assumption and sex was found.

Painkillers were taken for $4.34 \pm 4.39$ days and the main types were paracetamol and NSAIDs.

Among the 35 patients, 2 had undergone a previous contralateral thoracotomy and confirmed a less postoperative pain after U-VATS approach.

The cosmetic result was good or excellent for the $100 \%$ of the series $(2.44 \pm 0.51$, evaluated on a scale from 1 to 3 points). No wound infection was recorded. Two patients (5.7\%) complained medium level paresthesia of the wound 7 days after the operation, spontaneously resolved during the follow-up.

\section{Discussion}

The efficacy of VATS in the treatment of pleural empyema has been proven recently. In fact, traditionally stages II and III have been managed by thoracotomy that gives the possibility to perform an extended decortication safely. 
Table 3 Improvement in phlogosis indexes and lung re-expansion after uniportal-VATS toilette

\begin{tabular}{lccc}
\hline Variable & Preoperative period & Post-operative period & $P$ \\
\hline Re-expansion of the lung & $8 / 35(22.8 \%)$ & $33 / 35(94.3 \%)$ & $<, 0.005^{\star}$ \\
White blood cell count & $14,300 \pm 7,445$ & $7,464 \pm 2,570$ & $<<0.005^{\star}$ \\
C-reactive protein $(<5 \mathrm{mg} / \mathrm{L})$ & $173.32 \pm 99.62$ & $84.61 \pm 67.85$ & $<<0.005^{\star}$ \\
Procalcitonin $(<0.5 \mathrm{ng} / \mathrm{mL})$ & $1.22 \pm 2.19$ & $0.35 \pm 0.60$ & 0.4 \\
\hline
\end{tabular}

*, $\mathrm{P}<0.05$. VATS, video-assisted thoracic surgery.

Table 4 Postoperative outcomes

\begin{tabular}{lc}
\hline Variables & Data \\
\hline First postoperative day pain (VAS scale) & $1.83 \pm 1.53$ \\
Mean duration of pain (days) & $2.63 \pm 2.23$ \\
Pain after chest tube removal (yes) & $3(8.6 \%)$ \\
Pain after chest tube removal (VAS scale) & $0.33 \pm 0.92$ \\
Cosmetic result (1 to 3 points) & $2.44 \pm 0.51$ \\
Postoperative paresthesia & $2(5.7 \%)$ \\
Wound infections & $0(0 \%)$ \\
\hline
\end{tabular}

VAS, Visual Analogue Scale.

In 2001, Waller and colleagues (7) concluded that in the management of Stage III empyema, VATS had been shown to be as effective as open decortication in a significant proportion of patients, with all the benefits of minimally invasive approach (in terms of pain and hospitalization). Conversion rate up to $40 \%$ was correlated to chronic stage of empyema but above all to surgeon's expertise with VATS, that requires a different learning curve.

Several authors (8-13) reported better clinical outcomes of thoracoscopic decortications as compared to thoracotomy. A review of 14 publications that provided the best evidence among 68 others on the same topic, performed by Chambers et al. in 2010 (14), concluded that VATS was superior to open surgery in terms of postoperative pain, complications, morbidity, 30-day mortality and length of hospital stay and showed no difference in the relapse rate. A very recent meta-analysis (5) drew the same conclusions in favor of VATS, stating its effectiveness and superiority to open decortications, although, in some particular cases, VATS may need a conversion to thoracotomy. However, very few works evaluated the role of U-VATS approach in the treatment of pleural empyema. Nowadays, U-VATS represents one of the most innovative and less invasive thoracoscopic approach, involving a single intercostal space and potentially reducing post-operative pain (6).

In skilled hand thoracic surgeons, U-VATS allows a wide range of procedures, even the most complex in thoracic surgery, like sleeve lobectomies and bronchoplasties.

An increasing number of centers have been reporting their experience with U-VATS and often, at the beginning of their training, U-VATS debridement and decortications for empyema together with other small procedures (pleural biopsies and wedge resections) represent the biggest part of their series $(15,16)$.

Nevertheless, no paper has ever specifically evaluated the role of the technique in the treatment of different stage empyemas, assessing clinical outcomes, except a recent one by Bongiolatti and colleagues (17). They reported their experience with ultrasonographic preoperative staging followed by U-VATS decortication for pleural empyema. Thirty patients (47\%) underwent uniportal thoracoscopic pleural decortication and $34(53 \%)$ open decortication for empyema in stage II $(40 \%)$ or III $(60 \%)$ obtaining a complete debridement and decortication in all patients. U-VATS showed lower chest tube duration, hospital stay and complications compared to thoracotomy in their series. The authors concluded that U-VATS decortication is a safe and effective approach for well-selected patients based on a combination of clinical staging and imaging.

In accordance with our experience and expertise with U-VATS for major lung resections, we managed all our 35 cases in U-VATS as first choice, without a preoperative selection.

The patients in our series were quite complex, with an ASA score of 2, 3 or 4 in the $85.7 \%$ of cases and a stage IIIII empyema in the $88.6 \%$. A complete decortication was obtained in all patients without any need of further access 
or conversion and with a low rate of minor complications $(22.8 \%)$ and null 30-day mortality. Chest tube duration was similar to that reported in literature for traditional VATS and lower than that for thoracotomy (5). Hospital stay was longer in our series as compared to others reported in literature (17), probably because of the clinical conditions and comorbidities of our patients that required intensive care treatment in $40 \%$ of cases and in the $20 \%$ for more than 5 days. Postoperative results were very satisfactory in terms of clinical outcomes, postoperative pain and cosmetic results.

In fact, we evaluated the level of pain at the first postoperative day and after chest tube removal, as well as the incidence of paresthesias 7 days after the operation, and we found that the pain level was very low according to VAS scale and that it lasted for only few days $(2.63 \pm 2.23$ days $)$, with an almost total resolution after chest tube removal. Furthermore, no wound infection was recorded.

According to our experience, we believe that U-VATS has more advantages if compared with traditional VATS and Thoracotomy. It allows the surgeon to reach all sections of the pleural cavity with enough space for a wide range of surgical gestures, perfect visibility, safety and feasibility. Through this technique debridement and decortication result more accurate and safe, like in thoracotomy.

Furthermore, it has excellent postoperative outcomes in terms of less pain, fast recovery and cosmetic results.

Therefore, we consider U-VATS as a first choice also in 'stages II and III' empyemas when the necessary surgical expertise has been achieved, because it permits an easier performance of a more complete debridement and decortication versus traditional VATS, with a very low conversion rate risk.

The notable limits of our study are its retrospective nature, the small sample of patients and the lack of a comparison group (traditional VATS or thoracotomy). Further prospective and randomized studies (U-VATS $v s$. traditional VATS) on wider series are claimed for better defining the role of this minimally invasive technique.

U-VATS for the treatment of pleural empyema is safe and effective, even in stage II and III empyema, with relevant postoperative outcomes in terms of less pain, fast recovery and cosmetic results.

\section{Acknowledgements}

None.

\section{Footnote}

Conflicts of Interest: The authors have no conflicts of interest to declare.

Ethical Statement: This study was approved by the Institutional Review Board of Charité - Universitätsmedizin (Berlin) and Catholic University of Sacred Hearth (Rome).

\section{References}

1. Ferguson AD, Prescott RJ, Selkon JB, et al. The clinical course and management of thoracic empyema. QJM 1996;89:285-9.

2. Davies HE, Davies RJ, Davies CW. Management of pleural infection in adults: British Thoracic Society Pleural Disease Guideline 2010. Thorax 2010;65:ii41-53.

3. Scarci M, Abah U, Solli P, et al. EACTS expert consensus statement for surgical management of pleural empyema. Eur J Cardiothorac Surg 2015;48:642-53.

4. Finley C, Clifton J, Fitzgerald JM, et al. Empyema: an increasing concern in Canada. Can Respir J 2008;15:85-9.

5. Pan $\mathrm{H}, \mathrm{He} \mathrm{J}$, Shen J, et al. A meta-analysis of videoassisted thoracoscopic decortication versus open thoracotomy decortication for patients with empyema. J Thorac Dis 2017;9:2006-14.

6. Ismail M, Swierzy M, Nachira D, et al. Uniportal videoassisted thoracic surgery for major lung resections: pitfalls, tips and tricks. J Thorac Dis 2017;9:885-97.

7. Waller DA, Rengarajan A. Thoracoscopic decortication: a role for video-assisted surgery in chronic postpneumonic pleural empyema. Ann Thorac Surg 2001;71:1813-6.

8. Cardillo G, Carleo F, Carbone L, et al. Chronic postpneumonic pleural empyema: comparative merits of thoracoscopic versus open decortication. Eur J Cardiothorac Surg 2009;36:914-8.

9. Chan DT, Sihoe AD, Chan S, et al. Surgical treatment for empyema thoracis: is video-assisted thoracic surgery 'better' than thoracotomy? Ann Thorac Surg 2007;84:225-31.

10. Tong BC, Hanna J, Toloza EM, et al. Outcomes of videoassisted thoracoscopic decortication. Ann Thorac Surg 2010;89:220-5.

11. Casali C, Storelli ES, Di Prima E, et al. Longterm functional results after surgical treatment of parapneumonic thoracic empyema. Interact CardioVasc Thorac Surg 2009;9:74-8. 
12. Angelillo Mackinlay TA, Lyons GA, Chimondeguy DJ, et al. VATS debridement versus thoracotomy in the treatment of loculated postpneumonia empyema. Ann Thorac Surg 1996;61:1626-30.

13. Marks DJ, Fisk MD, Koo CY, et al. Thoracic empyema: a 12-year study from a UK tertiary cardiothoracic referral centre. PLoS One 2012;7:e30074.

14. Chambers A, Routledge T, Dunning J, et al. Is videoassisted thoracoscopic surgical decortication superior to open surgery in the management of adults with primary empyema? Interact Cardiovasc Thorac Surg

Cite this article as: Ismail $M$, Nachira D, Meacci E, Ferretti GM, Swierzy M, Englisch JP, Ossami Saidy RR, Faber S, Congedo MT, Chiappetta M, Petracca Ciavarella L, Margaritora S, Rueckert JC. Uniportal video-assisted thoracic surgery in the treatment of pleural empyema. $\mathrm{J}$ Thorac Dis 2018;10(Suppl 31):S3696-S3703. doi: 10.21037/jtd.2018.05.10
2010;11:171-7..

15. Roubelakis A, Modi A, Holman M, et al. Uniportal videoassisted thoracic surgery: the lesser invasive thoracic surgery. Asian Cardiovasc Thorac Ann 2014;22:72-6.

16. Abu Akar F, Gonzalez-Rivas D, Ismail M, et al. Uniportal video-assisted thoracic surgery: the Middle East experience. J Thorac Dis 2017;9:871-7.

17. Bongiolatti S, Voltolini L, Borgianni S, et al. Uniportal thoracoscopic decortication for pleural empyema and therole of ultrasonographic preoperative staging. Interact CardioVasc Thorac Surg 2017;24:560-6. 